\title{
Back Mutation of Leucine-Requiring Auxotrophs of Salmonella typhimurium Induced by Diethylsulphate
}

\author{
By A. RONEN* \\ Department of Biology, University of California, San Diego, La Jolla, California, \\ U.S.A.
}

(Received 21 April 1964)

\begin{abstract}
SUMMARY
Ten leucine-requiring mutants of Salmonella typhimurium were induced to revert with the alkylating agent, diethylsulphate (DES). The pattern of reversion was studied by following the appearance of leucine-independent colonies on minimal plates, and of turbid (revertant) tubes among tubes containing $10^{5}-10^{6}$ treated bacteria in minimal medium. Five mutants gave high $\left(0 \cdot 6-5 \times 10^{-5}\right)$ frequencies of reversion. The other five gave lower $\left(5 \times 10^{-8}-1 \cdot 3 \times 10^{-6}\right)$ frequencies immediately after plating on minimal agar or dilution in minimal medium, but reversion continued to take place until a high frequency was attained. This delayed mutation was found to be independent of cell metabolism. The possibility is discussed that the different patterns of reversion reflect alkylation of different base pairs. It is suggested that mutants having guanine: cytosine $(\mathrm{G}: \mathrm{C})$ base pairs at the mutant site revert mainly by pairing errors made by alkylated guanine. Mutants with adenine : thymine (A:T) sites are assumed to revert through the production of apurinic gaps, formed by the hydrolysis of ethyladenine.
\end{abstract}

\section{INTRODUCTION}

It has been proposed that ethylating agents exert their mutagenic effects mainly through the production of 7 -ethylguanine (EG) in the DNA molecule (Bautz \& Freese, 1960). The other major product of alkylation, 3-ethyladenine (EA), is assumed to have a lesser role in ethylation-induced mutagenesis (Brooks \& Lawley, 1962; Pal, 1962; Krieg, $1963 a, b)$. Ethylation-induced mutation of phage exhibits a pattern of delayed mutation, in which mutant subclones appear to be formed after normal non-mutant gene copies have been made (Green \& Krieg, 1961). Two interpretations of this phenomenon have been advanced, based on different base-pairing properties of ionized or tautomerized EG, or based on hydrolysis of EG (Green \& Kreig, 1961; Freese, 1963; Krieg, 1963a). Experiments to test the latter interpretation did not support it and favoured the pairing-error interpretation of the delayed mutant subclones (Löbbecke \& Krieg, cited in Krieg, 1963b).

In experiments with bacteria it is common practice to count revertant colonies at an early time (36-48 hr), to avoid counting slow-growing colonies which may be due to suppressor mutations (Margolin \& Mukai, 1961). Yet it is common experience to find a continuous appearance of new colonies on the selective plates after plating bacteria treated with alkylating agents.

Two years ago an experiment was designed by Professor F. J. Ryan and the present author to study diethylsulphate (DES)-induced recombination in Salmonella

* Present address: Laboratory of Genetics, The Hebrew University, Jerusalem, Israel. 
typhimurium. The results were indecisive because of a continuous reversion of some of the markers, although the treated bacteria were kept in buffer. This finding could not be accounted for by selection. Somewhat similar results were found by Strauss \& Okubo (1960), who found increased reversion from auxotrophy to prototrophy upon incubation of DES-treated Escherichia coli at an elevated temperature before selective plating was done. In the present paper, a study of the pattern of mutant appearance after DES treatment is described. It shows that late appearance of revertant colonies after DES treatment is indicative of delayed mutation rather than slowly growing suppressor mutations, and is not due to a general 'physiological damage' of alkylation. It further shows that the different mutations can be grouped in two classes-delaying and non-delaying ones-which can be correlated with the pattern of mutation found after treatment with some base analogues.

\section{METHODS}

Organisms used. Leucine-requiring mutants of Salmonella typhimurium strain LT 2 were used (we are indebted to Dr P. Margolin for these mutants) and are among 42 studied by Margolin \& Mukai (1961) which were shown to be revertible by both diethylsulphate and 2-amino-purine (AP). They fell into two groups with respect to AP-induced reversion: those which showed a higher frequency of induced mutation when several cell divisions occurred after treatment (division-dependent, DD), and those whose frequency of induced reversion was not dependent on further cell division (division-independent, DI).

Media. Liquid minimal medium (MM) used in reversion experiments contained (g./l.): $10.5, \mathrm{~K}_{2} \mathrm{HPO}_{4} ; 4.5, \mathrm{KH}_{2} \mathrm{PO}_{4} ; 1.0,\left(\mathrm{NH}_{4}\right)_{2} \mathrm{SO}_{4} ; 0.98, \mathrm{Na}$-citrate; $8.0, \mathrm{NaCl}$; 2.0, glucose; $1 \mathrm{ml}$. $\mathrm{M}-\mathrm{MgSO}_{4}$; water to $1 \mathrm{l}$. Minimal agar (MA) contained, in addition, 20 g. agar/l. The same minimal agar, supplemented with $25 \mu \mathrm{g}$. leucine/ml., was used for viable counts. Tryptone broth contained (g./l.): 10, Bacto tryptone; 9, $\mathrm{NaCl} ; 2$, glucose. The buffer used for treatment as well as post-treatment incubation contained (g./l.): 5·8, $\mathrm{Na}_{2} \mathrm{HPO}_{4} ; 3, \mathrm{KH}_{2} \mathrm{PO}_{4} ; 1, \mathrm{NH}_{4} \mathrm{Cl} ; 8, \mathrm{NaCl}$; and $1 \mathrm{ml}$. $\mathrm{M}-\mathrm{MgSO}_{4}$; water to 11 .

Treatment. Overnight cultures grown in $100 \mathrm{ml}$. tryptone broth were washed twice and resuspended in $10 \mathrm{ml}$. buffer. After further incubation for about $3 \mathrm{hr}$ at $37^{\circ}$, diethylsulphate was added to $5 \mathrm{ml}$. of suspension. The treatment mixture was shaken at $37^{\circ}$ on a shaker. Treatment was stopped by diluting $0.05 \mathrm{ml}$. of the mixture into $5 \mathrm{ml}$. buffer supplemented with $\mathrm{Na}_{2} \mathrm{~S}_{2} \mathrm{O}_{3}$ to a final concentration of $0.04 \mathrm{M}$. Whenever large numbers of treated bacteria were needed, $1.0 \mathrm{ml}$. of the treated suspension was diluted in $100 \mathrm{ml}$. of buffer supplemented with $\mathrm{Na}_{2} \mathrm{~S}_{2} \mathrm{O}_{3}$. After incubation at $37^{\circ}$ for $1 \mathrm{hr}$, the bacteria were collected by centrifugation and resuspended in $5 \mathrm{ml}$. buffer containing $0.04 \mathrm{M}-\mathrm{Na}_{2} \mathrm{~S}_{2} \mathrm{O}_{3}$.

Plating experiments. Plates of minimal agar (MA) seeded with $10^{6}-10^{9}$ bacteria were incubated at $37^{\circ}$, and revertant colonies marked and counted. In each experiment, a $36 \mathrm{hr}$ colony from a control MA plate (seeded with untreated bacteria) was chosen as a 'reference colony' (and the plate kept in a refrigerator). Revertant colonies were counted only after attaining the size of that colony.

Tube experiments. For tube experiments, treated bacteria were further diluted in chilled liquid minimal medium (MM) and distributed in small tubes. The tubes were incubated at $37^{\circ}$ without aeration and examined routinely for the appearance 
of turbidity caused by overgrowth of revertants. Whenever it was necessary to follow the optical density in those tubes, $4 \mathrm{ml}$. samples were distributed; otherwise, each tube contained $2 \mathrm{ml}$. A Bausch and Lomb 'Spectronic 20' spectrophotometer was used for readings of optical density at $540 \mathrm{~m} \mu$.

\section{RESULTS \\ Alkylation of mutant leu-524}

The effects of diethylsulphate (DES) treatment on viability and mutation in the Salmonella typhimurium mutant, leu-524, are shown in Fig. 1. Samples $(5 \mathrm{ml}$.) of buffer suspension of bacteria were treated with different DES concentrations for $20 \mathrm{~min}$., or with $0.04 \mathrm{ml}$. (final concentration of $0.06 \mathrm{M}$ ) of DES for various lengths of time. When the whole range of diethylsulphate concentrations used was drawn so as to match the whole range of treatment time, the two curves closely resembled one another within the ranges of times and doses shown; they deviated, however, with more severe treatments. This was because of rapid decomposition of DES in aqueous solution and because, with higher concentrations of DES, the oily DES drops in the treatment suspension become very large and remain attached to the bottom of the tube. A $20 \mathrm{~min}$. treatment with $0.04 \mathrm{ml}$. DES $/ 5 \mathrm{ml}$. bacterial suspension was arbitrarily chosen as standard treatment in further experiments.

The reversion data shown in Fig. 1 were obtained by treating $5 \mathrm{ml}$. bacterial suspension with different concentrations of DES for 10 and $20 \mathrm{~min}$. Colonies growing on minimal medium agar (MA) were counted after 5 days. After that time, there was little change in colony count from day to day. One can, however, count the colonies on the same reversion plates every day, and plot the additive numbers against time. When this was done with mutant leu-524, the results shown in Fig. 2 were obtained. Spontaneous revertants form colonies which reach the size of the reference colony on MA after $30-40 \mathrm{hr}$, and there is little or no increase in their numbers after $60 \mathrm{hr}$. DES-induced revertant colonies lag behind the spontaneous colonies, and their number continues to increase considerably for another $96 \mathrm{hr}$. $A$ priori, this increase in colony count could be accounted for by some physiological variability, slow-growing suppressor mutants, or delay in back mutation of leu-524 itself. On the other hand, the possibility exists that the DES curves in Fig. 2 finally levelled off only because of environmental changes that took place on the plates while they were being incubated (such as bacteria becoming devoid of nutrients as the plates became too dry).

To test these possibilities, the following experiment was made. Treated bacteria of strain leu-524 were diluted in MM to approximately $10^{5}$ bacteria/ml.; $2 \mathrm{ml}$. samples were distributed in small tubes and allowed to stand at $37^{\circ}$ without aeration. Control bacteria were diluted in $\mathrm{MM}$ to about $10^{7}$ bacteria/ml. (very slight turbidity). The results are shown in Fig. 3. Most of the control tubes remained clear for about 3 weeks. However, some tubes became turbid because of the overgrowth of leu ${ }^{+}$revertants. Pre-existing mutants required, under those conditions, about $48 \mathrm{hr}$ for full overgrowth. After that time, no more mutants were found. (The rate of spontaneous reversion in leu-524 is too low to become manifest at these conditions.) In the treated series, the number of turbid tubes continued to increase, although at a decreasing rate, throughout the experiment. 
One can estimate, for any given time, the mean number $(m)$ of mutant bacteria per tube (Ryan, 1955). After a time sufficient for full overgrowth (as made manifest by increase of turbidity), $m$ represents the average number of pre-existing mutants originally distributed in the tubes. Thereafter, $m$ also includes new mutants which

MI. diethylsulphate added to $5 \mathrm{ml}$. buffer (20 min. exposure)

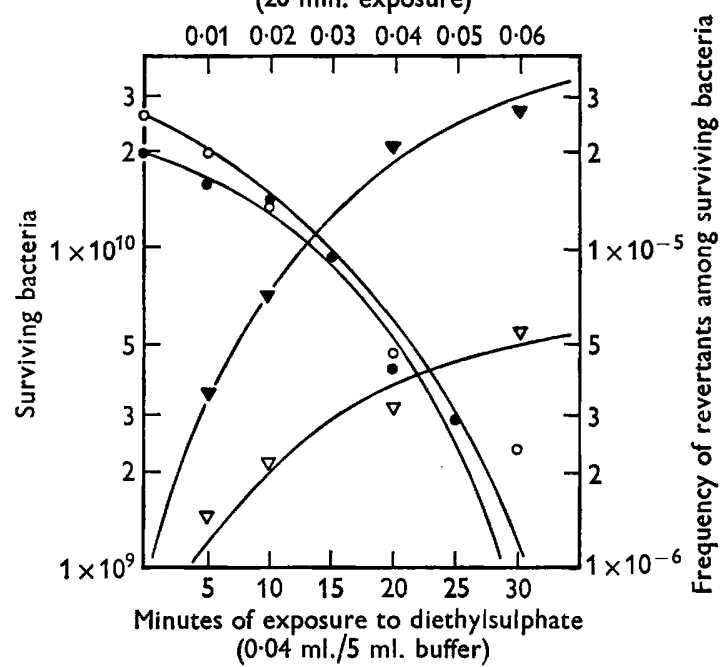

Fig. 1

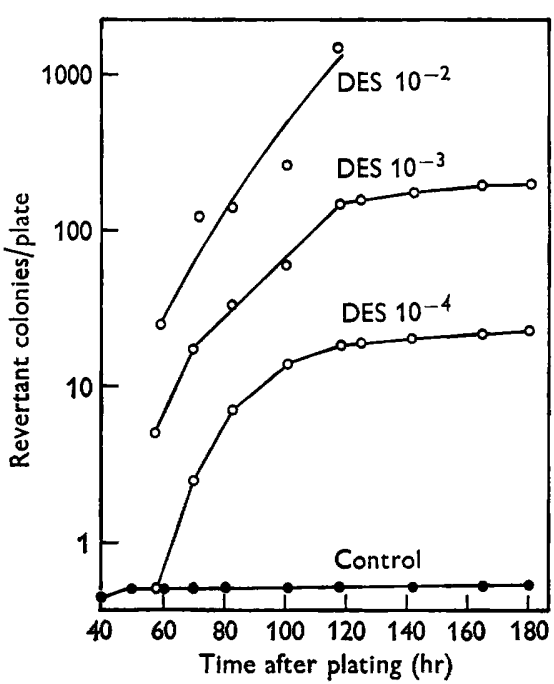

Fig. 2

Fig. 1. Viability and mutation frequency after various treatments with diethylsulphate. 0 - Viability after treatment with $0.04 \mathrm{ml}$. diethylsulphate $/ 5 \mathrm{ml}$. bacterial suspension for various lengths of time. $O=$ Viability after treatment with various concentrations of diethylsulphate for $20 \mathrm{~min}$. $\nabla=$ The frequency of revertants among surviving bacteria after $20 \mathrm{~min}$. treatment with various concentrations of diethylsulphate. $\nabla=$ The frequency of revertants among surviving bacteria after $10 \mathrm{~min}$. treatment with various concentrations of diethylsulphate.

Fig. 2. The number of revertant colonies growing on minimal medium agar, as recorded at different times after plating. A culture of $c$. $10^{10}$ leu-524 bacteria/ml. was treated with diethylsulphate and plated at final dilution of $10^{-2}, 10^{-8}$ and $10^{-4}$. Control bacteria were plated undiluted.

originated after the bacteria were distributed in the tubes. $m$ is estimated by determining the fraction of clear tubes among all the tubes $\left(\boldsymbol{P}_{0}\right)$, and applying it to the zero term of the Poisson distribution:

$$
P_{0}=e^{-m}
$$

In Fig. 3, typical $m$ curves for control and DES-treated leu-524 bacteria are shown. In the control $m$ increased from 30 to $48 \mathrm{hr}$, after which there was no further increase. In the treated series $m$ increased continuously for more than $400 \mathrm{hr}$.

\section{'Suppressor' mutations}

Inasmuch as some suppressor mutations can be distinguished from 'true' reversion by the slow growth rate of suppressor mutants, mutant leu-524 gives, upon alkylation, mainly true revertants; only few slow-growing tubes were detected by routine measurements of optical density. Nor were many found in reconstruction 
experiments. In such experiments, single bacteria were isolated from tubes which had become turbid at different times in the main experiment. This was done by diluting them (so as to give, on the average, less than one cell per tube) into tubes
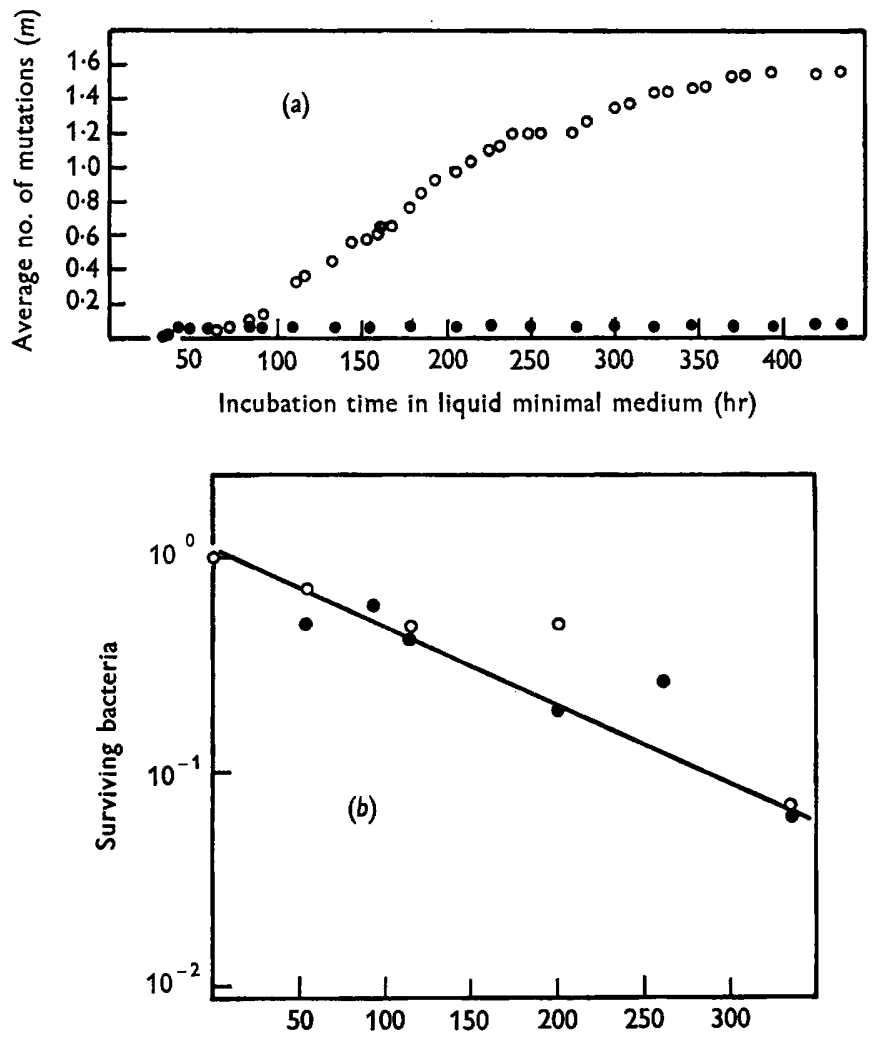

Incubation time in liquid minimal medium (hr)

Fig. 3. (a) The average number of mutations $(m)$ in control (closed circles) and diethylsulphate-treated (open circles) leu-524 bacteria. The total numbers of tubes were 285 and 492, respectively. (b) Survival of control (closed circles) and diethylsulphate-treated (open circles) in the above experiment.

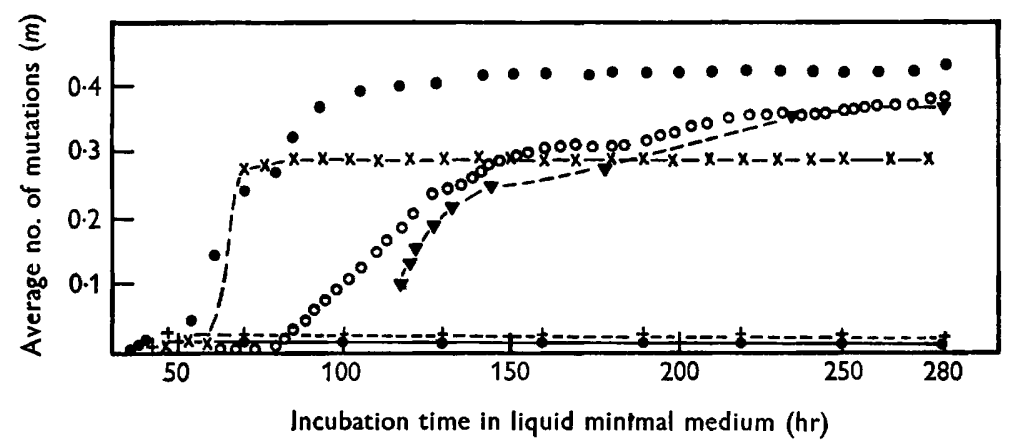

Fig. 4. The average number of mutations $(m)$ in control (bottom curves) 524, +---+; 48 and 122, $48, x-x ; 122,0 ; 524, O \bigcirc ; 524$ slow, $\nabla \longrightarrow \nabla$. 524 slow was the slow growing tubes from leu 524 . 
containing $2 \mathrm{ml}$. liquid minimal medium with or without a 'background' population of about $5 \times 10^{5}$ mutant (untreated) bacteria $/ \mathrm{ml}$. The time for overgrowth in each tube was measured. The same observation, namely, that 'early' and 'late' mutants grew at the same rate, was confirmed in later experiments by the use of the spectrophotometer: with few exceptions (considered as slow growers), it took 6-10 hr from the time tubes showed any measurable turbidity until they became fully turbid. However, by using the spectrophotometer it was possible to secure a sufficient number of slow-growing revertants. After $120 \mathrm{hr}$ from the time of dilution, the $m$ curve for slow growers followed closely the $m$ curve obtained with the normal growers (Fig. 4).

Table 1. Delayed mutation in various leucine mutants of Salmonella typhimurium

\begin{tabular}{|c|c|c|c|c|c|c|}
\hline \multirow[b]{2}{*}{ Mutant } & \multicolumn{2}{|c|}{$\begin{array}{c}\text { Frequency of } \\
\text { spontaneous mutation }\end{array}$} & \multicolumn{2}{|c|}{$\begin{array}{c}\text { Frequency of } \\
\text { diethylsulphate-induced } \\
\text { mutation }\end{array}$} & \multirow{2}{*}{$\begin{array}{c}\text { Division- } \\
\text { dependence* }\end{array}$} & \multirow{2}{*}{$\begin{array}{c}\text { Delayed } \\
\text { mutation }\end{array}$} \\
\hline & At $48 \mathrm{hr}$ & After 7 days & At $48 \mathrm{hr}$ & After 7 days & & \\
\hline leu-10 & $1.7 \times 10^{-9}$ & $2.3 \times 10^{-9}$ & $8.8 \times 10^{-8}$ & $>10^{-4}$ & + & + \\
\hline$l e u-25$ & $3.0 \times 10^{-9}$ & $5.0 \times 10^{-9}$ & $5.0 \times 10^{-8}$ & $2.8 \times 10^{-6}$ & + & + \\
\hline leu-34 & $4.6 \times 10^{-9}$ & $6.3 \times 10^{-9}$ & $1.4 \times 10^{-7}$ & $8.9 \times 10^{-5}$ & + & + \\
\hline leu-44 & $1.2 \times 10^{-2}$ & $2.3 \times 10^{-9}$ & $2.0 \times 10^{-5}$ & $5 \cdot 1 \times 10^{-5}$ & - & - \\
\hline leu-48 & $1.0 \times 10^{-\theta}$ & $2.0 \times 10^{-9}$ & $2.8 \times 10^{-5}$ & $3.9 \times 10^{-5}$ & - & - \\
\hline leu-122 & $4.2 \times 10^{-9}$ & $7.8 \times 10^{-9}$ & $5.4 \times 10^{-6}$ & $5.9 \times 10^{-6}$ & - & - \\
\hline leu-127 & $1.3 \times 10^{-9}$ & $4.5 \times 10^{-9}$ & $3.8 \times 10^{-5}$ & $4.0 \times 10^{-5}$ & - & - \\
\hline leu-130 & $4.3 \times 10^{-9}$ & $8.3 \times 10^{-9}$ & $2.4 \times 10^{-5}$ & $2.5 \times 10^{-5}$ & - & - \\
\hline leu-140 & $1.7 \times 10^{-8}$ & $3.9 \times 10^{-8}$ & $6.5 \times 10^{-7}$ & $1.3 \times 10^{-5}$ & + & + \\
\hline leu-524 & $6.4 \times 10^{-10}$ & $9.5 \times 10^{-10}$ & $1.3 \times 10^{-6}$ & $2 \cdot 4 \times 10^{-5}$ & + & + \\
\hline
\end{tabular}

* As observed by Margolin \& Mukai (1961): $+=$ division-dependent; - = divisionindependent.

Experiments with other leu mutants

Having thus established that delayed mutation in mutant leu-524 was not an artifact caused by slow growth, it became desirable to see whether other leucine mutants would show the same phenomenon after treatment with diethylsulphate (DES), or whether the delayed mutation was peculiar to mutant leu-524. Nine other DES-revertible leu mutants were selected for this purpose, on the basis of their patterns of reversion with 2-aminopurine (Margolin \& Mukai, 1961) (Table 1, column 6). Buffer suspensions of these mutants were treated with diethylsulphate as described for mutant leu-524, and the numbers of revertant colonies were recorded repeatedly.

The results obtained with all 10 mutants are summarized in Table 1 and in Fig. 5. It can be clearly seen that all mutants did not show the delay effect. Mutants 44 , $48,122,127$ and 130 (namely, all division-independent mutants), after the appearance of the first burst of revertant colonies, show no further increase in the number of revertant colonies. On the other hand, all five division-dependent mutants did show such an increase for more than a week.

\section{Delayed mutation on minimal medium agar and in buffer}

A question arises about the relation between diethylsulphate-induced mutation and division-dependence after treatment with 2-aminopurine. To separate the time effect from the cell-replication effect, treated bacteria were kept in buffer before 
plating was done on minimal medium agar. The results are shown in Fig. 6. Leu-524 bacteria showed about a 40-fold increase in the number of revertant colonies over the first burst, when plated immediately after treatment. After incubation in buffer for $72 \mathrm{hr}$ there was less than a 1.5-fold increase in the number of revertant colonies (Fig. 6a). This was even more pronounced in another experiment (Fig. 6b) where treated bacteria were kept in buffer for $140 \mathrm{hr}$ before a tube test was made.



Fig. 5


Post-treatment incubation in minimal liquid medium (hr)

Fig. 6

Fig. 5. Frequency of revertant colonies of the various mutants on minimal medium agar, as recorded at different times after plating. The frequencies of induced reversion of all 10 mutants are shown (10 upper curves). To make the figure more legible, only some of the control curves are shown. The others fall within the same limits and show the same pattern. The figures for leu-524, are magnified ten times.

Fig. 6. (a) Reversion frequency of leu-524 on minimal medium agar, as recorded at different times after plating. $0=$ Cells plated immediately after treatment. $72=$ Cells plated after $72 \mathrm{hr}$ of post-treatment incubation in buffer. (b) The average number of mutants $(m)$ among diethylsulphate-treated leu-524, bacteria in a tube experiment. -0 cells distributed immediately; $\mathrm{O}-\mathrm{O}=140$ cells distributed after $140 \mathrm{hr}$ of post-treatment incubation in buffer.

In contrast with the gradual increase in the $m$ curve obtained with bacteria which were distributed immediately after treatment, the rise in $m$ values for the bacteria which had been kept in buffer was rather abrupt, with no further increase. In both cases, however, the actual frequency of revertants was the same whether or not the bacteria had been aged in buffer before selection. 


\section{DISCUSSION}

Margolin \& Mukai (1961) studied 2-aminopurine-induced reversion of some 42 leucine-requiring mutants of Salmonella typhimurium, including 9 of the mutants used in the present work. They were able to demonstrate a distinction between continuous (division-dependent) and a single burst (division-independent) mutation. Both theoretical considerations (Freese, 1959) and the patterns of mutation after 2-aminopurine and 5-bromouracil found in Escherichia coli (Strelzoff, 1962) strongly suggest that the division-dependent mutants, $10,25,34,140$ and 524 have adeninethymine base pairs at their mutant sites, while mutants $44,48,122,127$ and 130 have guanine-cytosine sites.

The present work shows that mutants of the two groups are diethylsulphate revertible and have different mutation patterns after diethylsulphate treatment. In spite of some degree of intra-group variability, mutants that are divisiondependent after 2-aminopurine treatment show delayed mutation after alkylation, while division-independent mutants do not. However, from the experiments with incubation in buffer it follows that this delay is dependent only upon time, and not on replication.

At present, little is known about mechanisms that can account for the different patterns of alkylation-induced mutation (see review by Krieg, 1963 b). Alkylation of native DNA was found to give mainly 7-alkylguanine and 3-alkyladenine (Lawley \& Brooks, 1963).

Krieg (1963a) found that $r$ II mutants of bacteriophage T4 that were highly revertible by ethyl methanesulphonate gave only a small fraction of their induced revertants when plated directly on selective host bacteria. The high frequency of induced revertants was found only when the treated phages were first allowed to reproduce in a non-selective host. (Neither procedure involved post-treatment storage of phage to promote depurination.) He attributed this phenomenon, and the delayed mutations occurring during replication (Green \& Krieg, 1961), to pairing errors of 7-ethylguanine. Other T4rII mutants, assumed to have adeninethymine pairs at their mutant sites, gave much lower or no ethyl methanesulphonateinduced reversion. Schwartz (1963), treating lac ${ }^{-}$mutants of Escherichia coli with ethyl methanesulphonate, failed to induce the reversion of ethyl methanesulphonateinduced mutants at a high frequency. He attributed it to the fact that ethyl methanesulphonateinduces mainly guanine-cytosine to adenine-thymine transitions. However, in the present study diethylsulphate was capable of reverting both guanine-cytosine and adenine-thymine mutant sites. Since diethylsulphate acts as a monofunctional ethylating agent like ethyl methanesulphonate, their mutagenicity should be expected to be similar.

Time-dependent delayed mutation in alkylation-induced bacteria is not necessarily identical with replication-dependent delay in phages. It is not clear, a priori, which of the alkylated bases should be implicated in interpreting the pattern of delayed mutation reported here. Nearly equal amounts of 7-ethylguanine and 3ethyladenine are released initially during post-treatment storage (Lawley \& Brookes, 1963). The apurinic gaps formed in this manner may account for the delayed mutations, by any of three mechanisms: (1) by taking in a 'wrong' base prior to replication (Ryan, Nakada \& Schneider, 1961); (2) by the incorporation of the 
latter opposite the gap, during replication; (3) by being capable of coding for the 'right' messenger RNA, thus initiating growth and replication. Such processes are quite speculative, but the idea of an apurinic gap leading to mutation is supported by the mutagenic effects of treatment at low $\mathrm{pH}$ values on phage (Freese, 1959), and of heat to bacteria (Greer \& Zamenhof, 1962).

It is also possible that the depurination-dependent process may be masked at guanine-cytosine sites, due to the 'erroneous' pairing of 7-ethylguanine with thymine (or uracil, in RNA synthesis) (Brookes \& Lawley, 1962). These hypothetical mechanisms are summarized in Fig. 7. Such a mechanism would be facilitated by

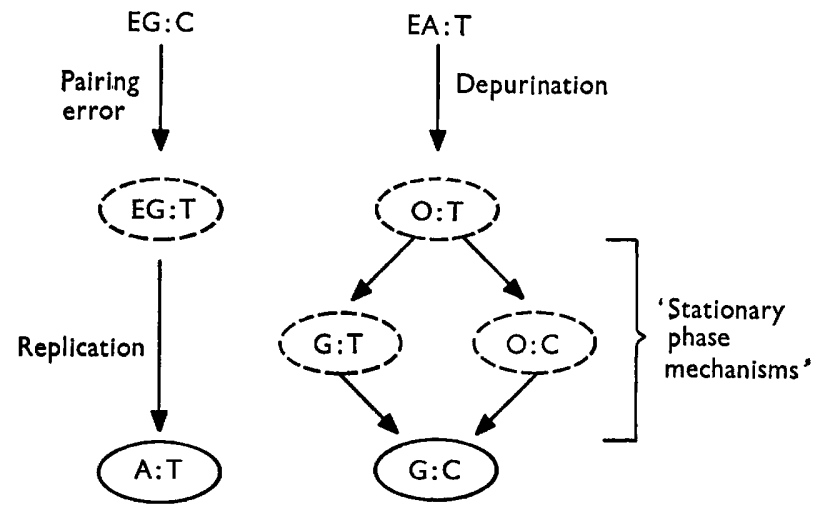

Fig. 7. Possible mechanisms of alkylation-induced reversion. Mutant 7-ethylguanine: cytosine (EG : C) sites are assumed to revert (immediately) through pairing errors during residual replication on minimal medium agar or in liquid minimal medium. 3-ethyladenine: thymine (EA:T) sites are assumed to revert through (delayed) depurination followed by insertion of guanine (G) in the apurinic gap, or by the polymerization of cytosine (C) opposite the gap. Of the other theoretical possibilities, delayed mutation through the production of apurinic gaps in EG: $\mathrm{C}$ sites is assumed to be masked by the process indicated here, while the ability of EA to pair with cytosine (Krieg, 1963a) has not been proven. $O=$ Apurinic gap. Base pairs encircled by a broken line indicate a possible partial (phenotypic) reversion. Base pairs encircled by a continuous line indicate complete reversion.

the technically uncontrolled residual replication of the bacteria upon resuspension in minimal medium. Whatever the mechanism of delayed mutation, further complications may be expected to stem from indirect effects, such as the possible influence of neighbouring bases in the DNA molecule, on base substitution. It is not unlikely that similar mechanisms underlie spontaneous mutation in the stationary phase (Ryan, 1959) and of diethylsulphate-induced mutation. More direct evidence and better understanding of the role of base turnover in stationary-phase mutation (Ryan et al. 1961) seem necessary for the interpretation of alkylationinduced mutagenesis. Experiments bearing on the uptake by bacteria of bases alkylated in vitro, and the pattern of consequent mutation, are underway now.

The author dedicates this paper to the memory of the late Professor Francis $\mathbf{J}$. Ryan, a teacher and a friend.

The author is indebted to Dr David R. Krieg for very helpful discussions and for his aid in the preparation of the manuscript. 
Parts of this work were carried out in the Department of Zoology, Columbia University, New York, and were supported by a P.H.S. grant to the late Professor F. J. Ryan.

\section{REFERENCES}

Bautz, E. \& Freese, E. (1960). On the mutagenic effect of alkylating agents. Proc. nat. Acad. Sci., Wash. 46, 1585.

Brooks, P. \& Lawley, P. D. (1962). The methylation of cytosine and cytidine. J. chem. Soc. p. 1348.

Freese, E. (1959). On the molecular explanation of spontaneous and induced mutations. Brookhaven Symp. Biol. 12, 63.

Freese, E. (1963). Molecular mechanism of mutation. In Molecular Genetics. Ed. by J. H. Taylor, p. 207. New York and London: Academic Press.

GreEN, D. M. \& KRIEG, D. R. (1961). The delayed origin of mutants induced by exposure of extracellular phage T4 to ethyl methane sulfonate. Proc. nat. Acad. Sci., Wash. 47, 64.

Greer, S. \& Zamenhor, S. (1962). Studies on depurination of DNA by heat. J. molec. Biol. 4, 128.

KRIEG, D. R. (1963a). Ethyl methane sulfonate-induced reversion of bacteriophage T4rII mutants. Genetics, $48,561$.

KrIEG, D. R. (1963b). Specificity of chemical mutagenesis. In Progress in Nucleic Acid Research. Ed. by J. N. Davidson \& W. E. Cohn, vol. 2, p. 125. New York and London: Academic Press.

LAWLEy, P. D. \& Brooks, P. (1963). Further studies on the alkylation of nucleic acids and their constituent nucleotides. Biochem.J. 89, 127.

Margolin, P. \& MUKaI, F. (1961). The pattern of mutagen-induced back mutation in Salmonella typhimurium. Z. VererbLehre, 92, 330.

Pax, B. C. (1962). Studies on the alkylation of purines and pyrimidines. Biochemistry, 1, 558.

RYAN, F. J. (1955). Spontaneous mutation in nondividing bacteria. Genetics, 40, 726.

RYAN, F. J. (1959). Bacterial mutation in the stationary phase and the question of cell turnover. J.gen. Microbiol. 21, 530.

Ryan, F. J., Nakada, D. \& Schneider, M. J. (1961). Is DNA replication a necessary condition for spontaneous mutation? Z. VererbLehre, 92, 38.

Schwartz, N. M. (1963). Nature of ethyl methane sulfonate induced reversions of $\mathrm{lac}^{-}$ mutants of Escherichia coli. Genetics, 48, $135 \%$.

Strauss, B. \& OKubo, S. (1960). Protein synthesis and the induction of mutations in Escherichia coli by alkylating agents. J. Bact. 79, 464.

Strelzoff, E. (1962). DNA synthesis and induced mutations in the presence of 5bromouracil. II. Induction of mutations. Z. VererbLehre, 93, 301. 